\title{
Subject-specific computational prediction of the effects of elastic compression in the calf
}

\author{
Fanny Frauziols ${ }^{(1)(2)(3)}$, Pierre Badel ${ }^{(1)(2)(3)}$, Laurent

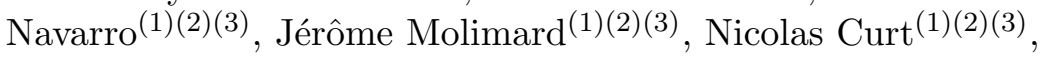 \\ Stéphane Avril ${ }^{(1)(2)(3)}$ \\ (1) Ecole Nationale Supérieure des Mines de Saint-Étienne, \\ CIS-EMSE, SAINBIOSE, F-42023 Saint-Étienne, France \\ (2) INSERM, U1059, F-42000 Saint-Étienne, France \\ (3) Université de Lyon, SAINBIOSE, F-42000 Saint-Étienne, France
}

\begin{abstract}
This chapter presents a quick and efficient approach to perform patient-specific Finite-Element (FE) predictions of the human calf response under elastic compression. The reconstruction of patient-specific material properties of soft tissues is based on two methodologies described extensively in two previous papers $[1,2]$. After a detailed description of the implementation of this model, results obtained on four volunteers are presented. The deformed geometry predicted by the FE model is in very good agreement with the MRI scan of the same leg under elastic compression. Eventually, it is shown that elastic compression always induces a partial vein closure but the magnitude of vein closure depends strongly on the position of the vein, on the shape of the leg and on the material properties of surrounding soft tissues.
\end{abstract}

\section{Introduction}

In this chapter, we are interested in the calf due to its major role in the venous blood circulation of our body. After a brief anatomical description, let us first introduce more in details the question of venous circulation and its possible dysfunctions.

\subsection{Anatomy}

The leg is the part of the lower limb between the joints of the ankle and the knee. It is composed of two long bones, tibia and fibula, which 
provide structural support. The distribution of soft tissue around the bones is uneven, with a thin part on the antero-medial side, a thicker part on the antero-lateral side and the thickest part on the entire rear side of the leg called calf.

In Figure 1, the soft tissues surrounding the two bones (tibia and fibula) of the leg are shown. The important components for our study are, from superficial to deep, skin, fat tissues under the skin, the fascia cruris and four muscle compartments. The veins are organized in three venous networks: superficial, deep and transverse [3].

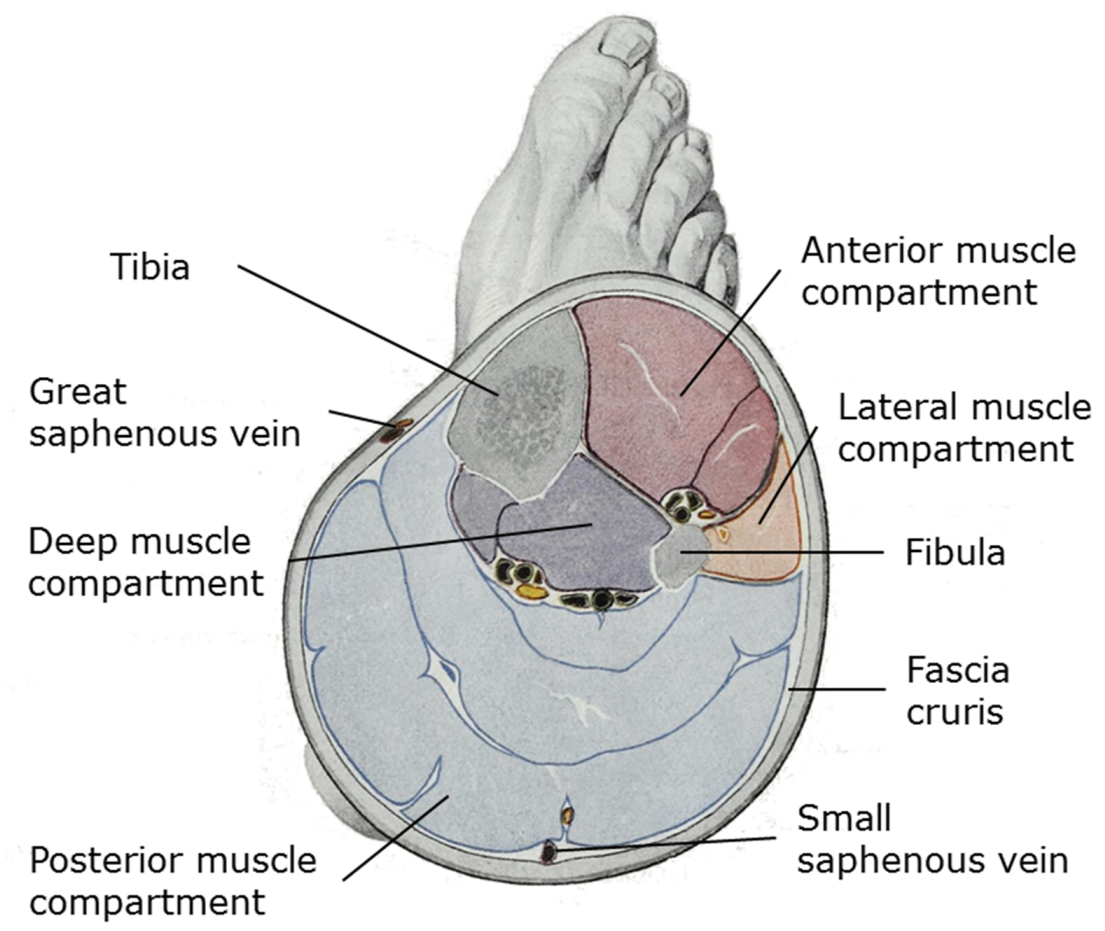

Figure 1 - Anatomy of the leg - Cross section of the right leg. The fascia cruris separates the superficial tissues, consisting of fat, skin and superficial veins, and the deep tissues, composed of four muscle compartments and deep veins. Adapted from [4]. 
Muscle tissues The leg is composed of four muscle compartments. A compartment includes one or several muscles and it is surrounded by a fascia. The composition of compartments and their physiological functions are explained below.

The anterior compartment is located between the fibula and the tibia. It is made of:

1. The anterior tibial muscle, responsible for dorsal flexion of the foot.

2. The extensor digitorum longus muscle, responsible for toe extension.

3. The extensor thumb muscle, responsible for thumb extension.

The lateral compartment is located on the side of the fibula. It is made of:

1. The short fibular muscle.

2. The long fibular muscle.

These two muscles are responsible for eversion of the foot.

The two posterior compartments (deep and superficial) are the largest, their role is essential in the gait.

The deep posterior compartment is made of:

1. the posterior tibial muscle, responsible for inversion of the foot and plantar flexion.

2. The flexor digitorum longus, responsible for toe flexion.

3. The flexor thumb, responsible for thumb flexion.

The posterior superficial compartment contains the triceps surae muscle. It is made of:

1. The gastrocnemius muscle.

2. The solear muscle, merging with the gastrocnemius muscle at the Achille tendon.

The triceps sural muscle acts on the knee and the ankle. It is responsible for knee flexion and plantar flexion. It has a major role in the venous blood return by squeezing the deep veins when it contracts.

\subsection{Fat tissues}

Adipose tissue is composed mostly of fat cells called adipocytes. Adipocytes store triglycerides to a cell size between $70 \mu \mathrm{m}$ and $120 \mu \mathrm{m}$. When this size is exceeded, the fat cell can not store fat and triggers the formation of a new cell. In a healthy subject, the adipose tissue is composed of 20 to 30 billion fat cells. This corresponds to $20-25 \%$ of the total mass for women 
and $15-20 \%$ for men.

We distinguish in the adipose tissue, white fat and brown fat (found mainly in newborns). White fat has a structural role by forming an adaptive medium to mechanical stress, for example by distributing the loads at the foot sole. Primarily located in the subcutaneous adipose tissue, it can integrate muscle compartments with age.

Fascia A fascia is a dense connective tissue surrounding muscle compartments. It essentially contains an extracellular matrix (made of elastic fibers and collagen fibers) surrounding fibroblasts. The fascia acts as a containing sheath which enhances muscle contraction. It may also have a proprioceptive role [5] similarly to the nervous system [6].

In the leg, two fascii should be distinguished: the fascia cruris and the fascia lata. There are also other fibrous membranes such as the membrane between the tibia and the fibula, and the intermuscular septa which is a fibrous membrane separating muscle groups). The fascia lata is the most superficial fascia. It is made of a layer of connective tissue located just below the skin, whereas the fascia cruris is thicker and denser. The two fascii are merged except at the location surrounding the two saphenous veins where the two fascii form a sheath around them [7]. This sheath seems to play a special role in reducing the risk of varicose veins as most of varicose veins are observed outside this sheath [8].

\subsection{Physiology of venous return}

Veins normally contain about $80 \%$ of the total volume of blood in the systemic vascular system. This role of blood stocking was considered for a long time as the only role of veins. Blood flow in the veins is however a topic of research that deserves as much attention as hemodynamics in the large arteries. Indeed, it has been estimated that more than $50 \%$ of women and $25 \%$ of men are affected by diseases related to chronic venous insuffiency (CVI), confirming that CVI is a major public health issue. CVI is a medical condition where the veins cannot pump enough oxygen-poor blood back to the heart. This may occur after deep vein thrombosis (DVT) or phlebitis. The latter is also a common complication in hospitalised patients. A serious outcome is pulmonary thrombo-embolism, which is implicated in $10 \%$ of all hospital deaths. The effects of CVI can grade from heavy legs and varicose veins to phlebetic lymphedema, chronic swelling of the legs and ankles, and increased risks of ulcers $[9,10]$. Although the effects of CVI are well known, the causes are rather complex, which makes them difficult to determine. 
It is important to dissociate in the venous system two types of veins:

1. the primary collecting veins which are passive, thin-walled reservoirs that are very distensible. Most are supra-fascial, surrounded by loosely bound alveolar and fatty tissue that is easily displaced. These suprafascial collecting veins can dilate to accommodate large volumes of blood with little increase in back pressure, so that the volume of blood sequestered within the venous system at any moment can vary by a factor of two or more without interfering with the normal function of the veins.

2. secondary veins or conduits which benefit of the outflow from primary collecting veins. They have thicker walls and are less distensible. Most of these veins are sub-fascial and are surrounded by tissues that are dense and tightly bound.

These veins may be grouped in 3 (sub)-systems:

1. the superficial venous system, which is a complicated and extremely variable network of interconnecting veins. Many superficial collecting veins deliver their blood into the great and small saphenous veins, which deliver most of their blood into the deep system through the sapheno-femoral junction SFJ and the sapheno-popliteal junction (SPJ).

2. the deep venous system which receives the blood before its way back to the right atrium of the heart.

3. the system of perforating veins which drains the blood from the superficial system to the deep veins. Venous valves prevent reflux of blood from the deep veins into the superficial system.

\subsection{The calf muscle pump}

The passage of blood upward from the feet against gravity depends on a complex array of valves and pumps. Muscle pumps of the calf and foot provide the motive force for venous return. This is frequently called the calf muscle pump or musculo-venous pump and is thought to function as the peripheral heart. Each segment of the calf muscle pump works in the same way. When the gastrocnemius and soleus muscles contract, they expel more than $60 \%$ of venous blood into the large popliteal vein. In the resting state, veins of the calf fill at 1 to 2 millimeters per second actively by compression of the sole of the foot and passively during muscle relaxation. It takes in average 70 seconds to refill $90 \%$ of the blood volume back into the calf and 31 seconds in average to restore hydrostatic pressure. However, these time durations can vary dramatically between individuals. Ambulatory venous pressure (AVP) is the 'gold standard' test of the efficiency of the calf 
musculo-venous pump. It is performed by placing a small needle into one of the veins on the back of the foot and connecting the needle to a blood pressure measurement machine. The test consists of three parts:

1. The subject is first asked to stand up and the standing venous pressure is measured.

2. The subject is asked to perform ten heel raise exercises to work the musculo-venous pump and the AVP is recorded.

3. The subject is asked to rest again in the standing position and the rate at which the ambulatory pressure returns to the standing pressure is measured, called the refilling time. In a normal subject the standing venous pressure is around $90 \mathrm{mmHg}$ (depending on their height). During exercise this should drop to around $30 \mathrm{mmHg}$ and after exercise this should only rise slowly over half a minute or so back to the standing pressure

\subsection{Venous dysfunction}

Venous dysfunction can result from primary muscle pump failure, from venous obstruction (thrombotic or nonthrombotic), or from venous valvular incompetence, which may be segmental or may involve the entire length of the vein. Venous return is impaired and it can trigger different possible abnormal AVP:

1. The pressure does not fall normally during exercise, which indicates that the calf pump is not working effectively.

2. The pressure rises rather than falls during exercise, which indicates that the deep veins are occluded.

3. The AVP returns to the standing pressure too quickly, which indicates reflux in either the deep or superficial veins due to absent or damaged valves or due to valves made incontinent because of dilation.

The effects of venous dysfunction can grade from heavy legs and varicose veins to phlebetic lymphedema, chronic swelling of the legs and ankles, and increased risks of ulcers.

\subsection{Treatments by compression therapy}

Compression stockings (CS), used as one of the important compression therapies, have been demonstrated to be an effective non-operative option to relieve symptoms associated with venous disorders in the human lower limb, such as reducing venous hypertension and improving venous blood return. Elastic compression is supposed to help the venous return, to decrease venous pressure, to prevent venous stasis and deterioration of venous 
walls, and to efficiently relieve aching and heavy legs. This simple, but very efficient therapy, is now also extended to recover from a sporting competition, or for instance as a prevention against the economy-class syndrome for transcontinental flights.

In case of DVT, medicinal anticoagulants, such as low molecular weight heparin, are often used in conjunction with CS. Intermittent pneumatic compression of the calf or foot can also be applied [11].

Scientific design of CS can not only enhance their medical functions, but also bring wearers comfort sensory perceptions. Comfort is an important issue. With the principle of graduated compression from the ankle to the knee, it is required to apply significant pressures at the ankle. Such high pressures at the ankle tend to be questioned and novel designs of compression stockings, with larger pressures at the level of the calf muscle, are now developed. Several medical studies based on the analysis of venous return with MRI and Doppler ultrasounds, have confirmed that large pressures at the calf are more efficient for treating moderate venous insufficiency [10].

\subsection{Objectives}

Now, the main limitation to develop new stocking products with targeted biomedical actions is that the biomechanical effects of compression therapy have not been fully elucidated. The role of computational models can be essential to address these issues.

Computational modelling was developed by several authors in the domain of venous deficiency and compression therapy. A review is presented in [10]. Previous biomechanical studies [12, 13, 14] highlighted the importance of inter-individual variations and the necessity to develop computational models on a patient-specific basis. This is the objective of the current chapter.

\section{Materials and Methods}

In a previous study [1], the material properties of superficial soft tissues were measured using ultrasound elastography (USE). In another previous study [2], the material properties of the deep soft tissues were identified by an inverse method. In this chapter we combine these two methods to define a biomechanical FE model customized in terms of geometry and mechanical properties for the study of the calf under elastic compression.

After outlining the key assumptions of this biomechanical model, its implementation is described in details. Then, after validation against MRI, 
results on 4 subjects are shown.

\subsection{Data acquisition}

In this biomechanical study of the leg under elastic compression, several experimental data are acquired. The same four volunteers $(3$ males and 1 female, mean age 27 years) than those of previous studies $[1,2]$ are considered. On each of them, MRI scan and USE data were acquired, whereas a local compression test was also carried out on the right hand side calf. The experimental protocol is explained in details in $[1,2]$.

\subsection{Assumptions about the anatomy}

The relevant components of the calf are, from superficial to deep: skin, fat, the fascia cruris, four muscle compartments and three venous networks (superficial, deep and transverse [3]).

Here, only two veins will be investigated: the small and the greater saphenous veins. Indeed, it appears that the elastic compression has a significant effect on these veins, whereas the effect on deep veins is rather negligible compared to the effect of muscle contraction $[14,15]$.

\subsection{Development of the FE model}

Geometry Both MRI and US data are acquired for the leg of each subject. The purpose of the MRI acquisition is to have all the internal details of the anatomy but the drawback is that the calf is deformed due to its contact with the platform in the scanner. The purpose of the US acquisition is threefold. First in B-mode and thanks to our system of image stitching [1], the internal details are acquired all around the calf up to a depth of about $3 \mathrm{~cm}$. Second, in USE mode, the elastic properties are measured up to a depth of about $3 \mathrm{~cm}$. Third, subjects are asked to achieve an isometric contraction equal to $30 \%$ of the maximum voluntary contraction and an image is also acquired in this state.

Registration between the MRI and US modalities is achieved by a rigid body transformation [1] using the bone contours (tibia and fibula) as landmarks (Figure 2).

Then, the overall geometry of the leg is defined from manual segmentation of ultrasound data, as the MRI geometry is deformed due to the contact of the calf with the platform in the scanner. The position and size of the saphenous veins (large and small) are also measured (Figure 3-B). Moreover, for each subject, two geometrical models are reconstructed: one 
in the inactive state of muscles and the other in the active state of muscles. The bones are segmented on MRI scan (Figure 3 - A) as the US image could not image to a sufficient depth.

As it is not possible to accurately measure the thickness of the vein walls with both modalities, the thickness is set at $1 / 10$ of the average diameter according to $[16,17]$.

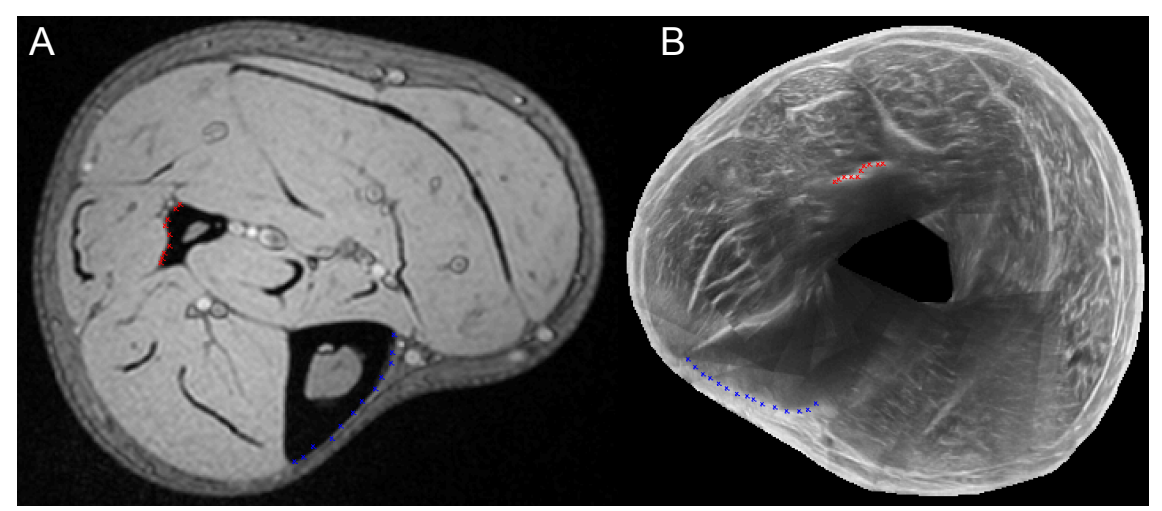

Figure 2 - Registration of two imaging modalities - The edges of the tibia (blue) and fibula (red) are identified on the MRI scan (A) and the ultrasonic reconstruction (B) to register the image by rigid body transformation.

The compression stocking The geometry of the CS is defined by a circle of radius, $R$, in its original state. The size is defined by the circumference at rest of the leg cross section imaged by MRI for a CS of class II, corresponding to an average applied pressure of $20 \mathrm{mmHg}$.

Meshing The contours segmented in the images are stored as a list of points in text files. An in-house developed Python script allowing to interact with the Abaqus/CAE environment is used to automate the construction of the FE mesh from text files. The meshes of the lower leg and of the compression stocking, created from the patient-specific geometry in Abaqus/CAE are presented in Figure 4.

Skin and compression stocking The skin and the CS are modeled by cable elements with linear interpolation (T2D2 in the Abaqus library). 


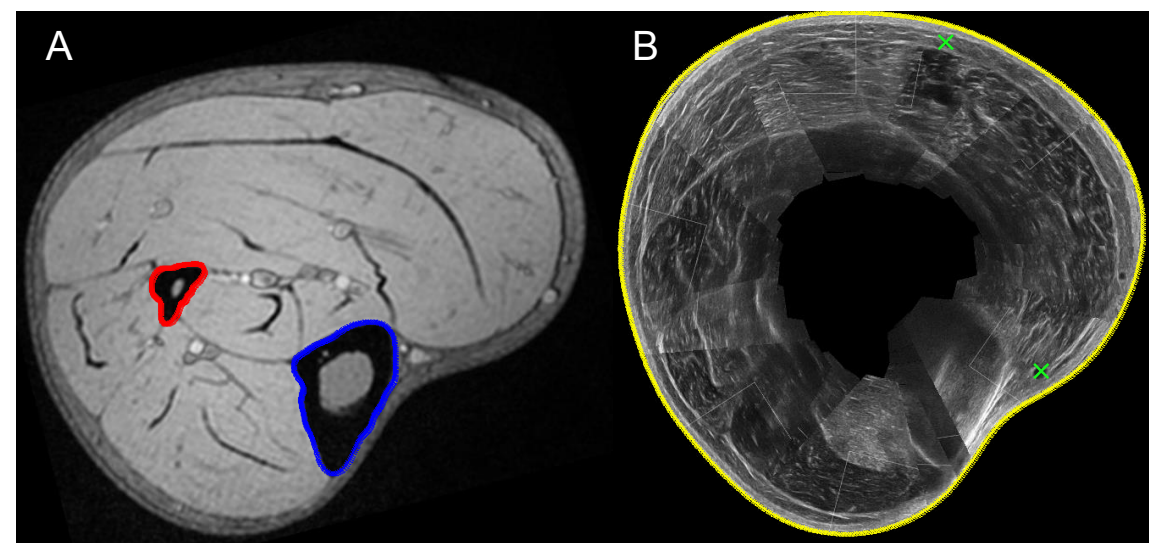

Figure 3 - Segmentation - On the transverse scan acquired by MRI (A), the edges of the tibia (blue) and fibula (red) are defined. On the US reconstruction (B), the overall geometry of the leg is segmented (yellow) and the position and size of the veins are identified (green).

Soft tissues Soft tissues are modeled by a hybrid version of continuous elements with linear interpolation in plane strain (CPE3H triangles and CPE4H quadrangles in the Abaqus library). The hybrid formulation can automatically manage incompressibility of biological soft tissues. Indeed, hybrid elements have an additional degree of freedom for the hydrostatic pressure.

Veins The mesh is refined near the vein (Figure 4) in order to have the same element size at the interface of veins and soft tissues. A previous study [15] permitted to determine the optimal mesh size of the vein elements for a good compromise between accuracy and computational cost. It was shown that 4 elements across the thickness of the vein is optimal.

\subsection{Material properties}

Superficial soft tissues The superficial soft tissues including adipose tissue (fat) and the fascia cruris are represented by a Neo-Hookean strain energy density function [18], which is defined by:

$$
\varphi=C_{10}\left(\bar{I}_{1}-3\right)+\frac{1}{D_{1}}\left(J^{e l}-1\right)^{2}
$$




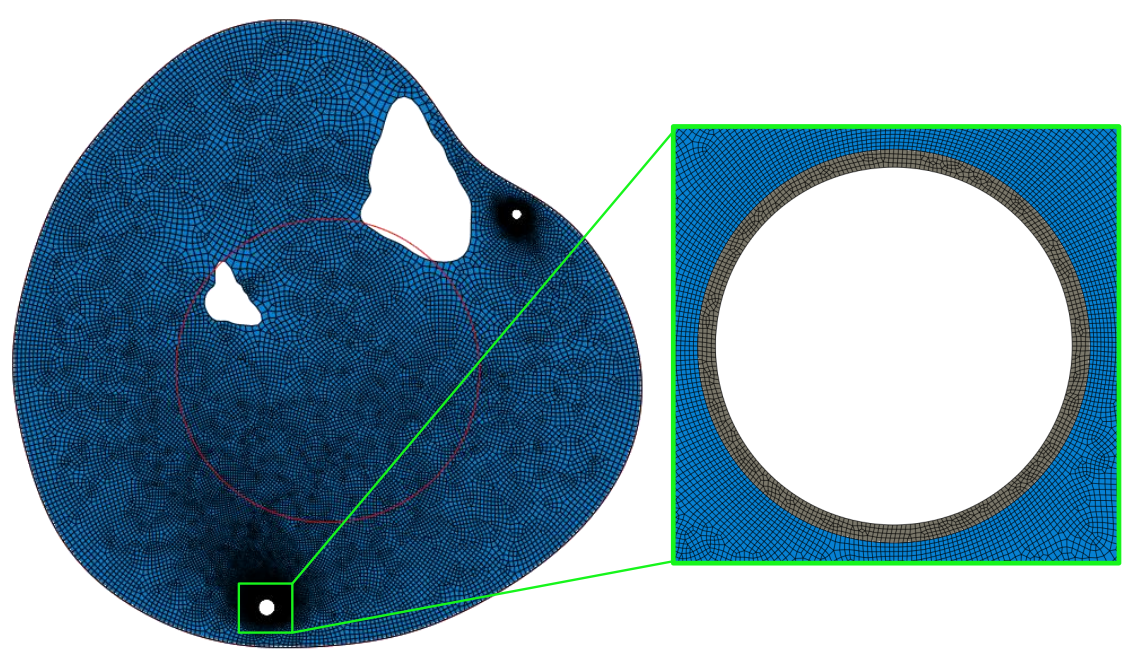

Figure 4 - Mesh of the lower leg and of the CS (red) in Abaqus/CAE generated from the patient-specific geometry.

With $\bar{I}_{1}$, first invariant of the left Cauchy-Green stretch tensor $(\overline{\mathbf{B}})$, derived from the isochoric part of the deformation gradient $\overline{\mathbf{F}}=J^{-\frac{1}{3}} \mathbf{F}\left(\bar{I}_{1}=\mathbf{I}: \overline{\mathbf{B}}\right)$; $J^{e l}$ is the Jacobian of the deformation $(J=\operatorname{det}(\mathbf{F})) ; C_{10}$ and $D_{1}$ are the material properties.

For each Gauss point of superficial soft tissues, elasticity data are available from USE [1] and material properties are assigned according to: $C_{10}=\frac{E}{6}$, where $E$ is the local elastic modulus measured with the USE technique. The value of the $D_{1}$ parameter is set according to literature data (Table 1).

Skin The probe used for the acquisition of USE data does not record specific values within the different layers of the skin ( $i e$ distinction not possible between the epidermis which is the outermost layer and the dermis below). This would require a higher frequency probe, and thus exclude the possibi- 
lity of acquiring deep images. So we model the skin as a whole monolayer and we assign generic material properties from the literature. These material properties may depend on the test type (torsion [19], tension [20] or suction [21]) and modeling assumptions (linear elasticity [22], viscoelasticity [23] or hyperelasticity). A Neo-Hookean hyperelastic behavior is assumed here. The values of material properties are given in Table 1.

Deep soft tissues Based on the results of a previous study [2], the deep soft tissues, comprising primarily the muscles, are represented by a polynomial strain energy density function of order 2 , which is defined by[18]:

$$
\varphi=C_{10}\left(\bar{I}_{1}-3\right)+C_{20}\left(\bar{I}_{1}-3\right)^{2}+\frac{1}{D_{1}}\left(J^{e l}-1\right)^{2}+\frac{1}{D_{2}}\left(J^{e l}-1\right)^{4}
$$

With $\bar{I}_{1}$, the first invariant of the left Cauchy-Green stretch tensor $(\overline{\mathbf{B}})$, derived from the isochoric part of the deformation gradient $\overline{\mathbf{F}}=J^{-\frac{1}{3}} \mathbf{F}$ $\left(\bar{I}_{1}=\mathbf{I}: \overline{\mathbf{B}}\right) ; J^{e l}$ is the Jacobian of the deformation $(J=\operatorname{det}(\mathbf{F})) ; C_{10}, C_{20}$ and $D_{1}, D_{2}$ are material properties.

Material properties $C_{10}$ et $C_{20}$ are identified with an inverse method coupling a 2D FE model with an in situ compression test applied onto the leg with a cylinder of diameter $30 \mathrm{~mm}$ [2].

Material parameters $D_{1}$ et $D_{2}$ are not identified in this study and are taken from literature (Table 1).

Material properties for the saphenous veins and the CS Veins and the CS are represented with linear elastic constitutive equations. Values of material properties ( $E$, Young's modulus and $\nu$, Poisson's ratio) are taken from literature and are reported in Table 1.

Assignment of material properties in the FE model Once the material properties of superficial and deep soft tissues are identified, they are assigned to each element of the FE mesh. For this, a Matlab script was written.

Boundary conditions Dirichlet boundary conditions are imposed on the contours of bones (clamping at the tibia et fibula).

\subsection{Modeling of elastic compression}

It is chosen to model the effect of the elastic compression with the CS directly by solving the problem of contact at the skin interface. For this, 
Table 1 - Material properties of each constituent of the model.

\begin{tabular}{lllc}
\hline Material & $\begin{array}{l}\text { Constitutive } \\
\text { behavior }\end{array}$ & Parameter & Origin \\
\hline Superficial & Hyperelastic & $C_{10}=\frac{E}{6} \mathrm{MPa}$ & US Elastography \\
soft tissues & Neo-Hookean & $D_{1}=22.5 \mathrm{MPa}^{-1}$ & {$[24]$} \\
\hline Deep & Hyperelastic & $C_{10}, C_{20}$ & Identified \\
soft tissues & polynomial & $D_{1}, D_{2}=28 \mathrm{MPa}^{-1}$ & {$[24]$} \\
\hline Skin & Hyperelastic & $C_{10}=0.1 \mathrm{MPa}$ & {$[25]$} \\
& Neo-Hookean & $D_{1}=0.14 \mathrm{MPa}^{-1}$ & \\
\hline Veins & Linear & $E=0.1 \mathrm{MPa}$ & {$[15]$} \\
& elastic & $\nu=0.49$ & {$[13]$} \\
\hline CS & Linear & $E=0.4 \mathrm{MPa}$ & \\
& elastic & $\nu=0.49$ & \\
\hline
\end{tabular}

several steps are required:

1. Swelling - a radial displacement equal to the local radius of the leg is imposed on nodes of the CS. This step 'inflates' the CS into a position just beyond the skin(Figure 5).

2. Contact - the radial displacement of the CS is left free and the contact with the skin is activated. During this step, only radial displacements are possible for the nodes of the CS.

3. Equilibrium - Sliding of the CS on the skin is now possible, permitting an adjustment of the CS around the leg. At the end of this step, equilibrium is reached.

\subsection{Contact definition}

Different contact models are available in Abaqus/Standard. These models define the discretization of the contact surfaces and algorithms governing normal and tangential behavior. The contacts chosen here are based on a previous study [15] having determined the best settings for a robust convergence of the model. These choices are summarized below.

A 'Surface - Surface' discretization is chosen. In this type of modeling, the contact is calculated on the entire surface unlike the discretization 'node - area' where the contact is calculated locally at each point of the surface. This provides a more even contact pressure and thus more representative of the reality. 

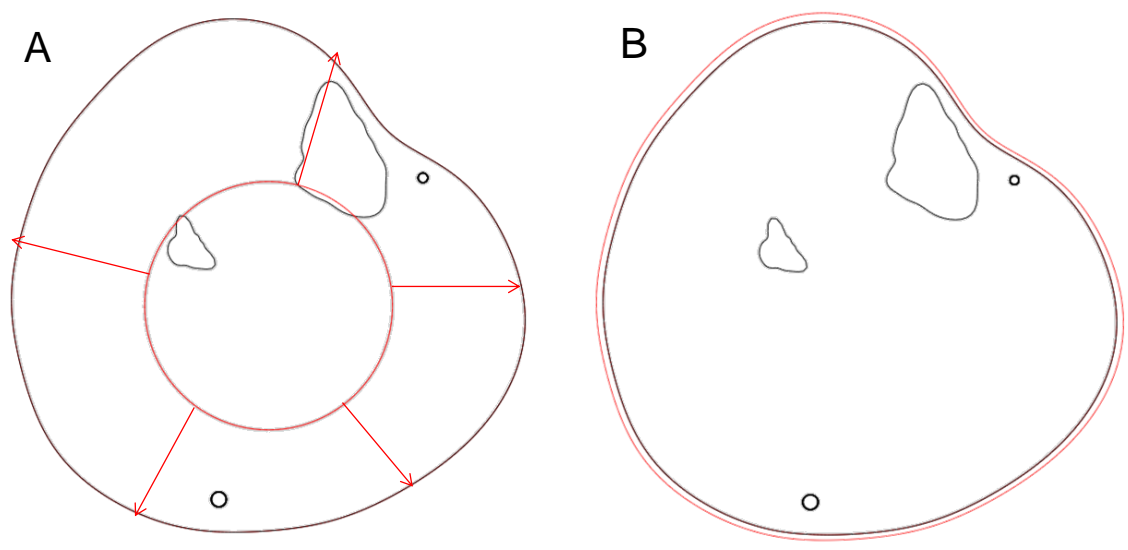

Figure 5 - Application of the contact pressure onto the leg - A radial displacement is imposed to the CS, equal to the local radius of the leg.

A normal contact condition is imposed to reflect the non-penetration of the CS in the leg. A tangential Coulomb dry friction is defined between superficial soft tissues and the CS with a coefficient of friction equal to 0.3 according to data from the literature [26]. A preliminary study showed that a change in the friction coefficient has little impact on the hydrostatic pressure distribution in soft tissues (Figure 6). A residual tension may be observed in the stocking when the friction coefficient is higher (Figure 7).

For the normal and tangential contact behavior, the penalty method is used. It allows easier convergence of the FE model. However, the Abaqus default penalty value is too small for our study. So to avoid over-penetration of the master area into the said slave surface, the penalty stiffness is manually adjusted.

Perfectly sticking contact is defined between the bone and the surrounding soft tissues. As bones (tibia and fibula) are clamped in the model, the surfaces of the soft tissues in contact with the bone are also clamped.

\subsection{Assessment of the FE model}

To evaluate the patient-specific FE model of the leg under elastic compression, the predicted deformations are compared to those obtained from an 

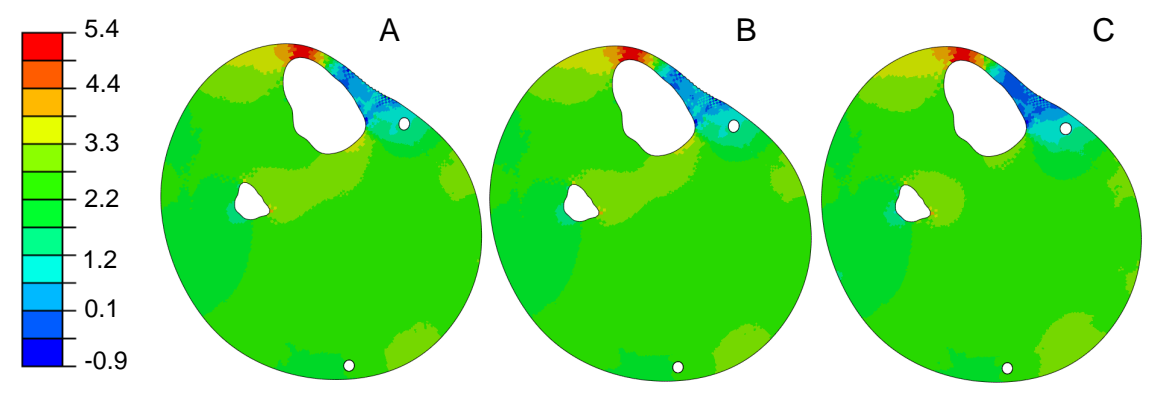

Figure 6 - Distribution of hydrostatic pressure in $\mathrm{kPa}$ within the soft tissues of the leg under elastic compression for a subject with an adjustment of the friction coefficient - Values of the friction coefficients: $\mathrm{A}=0.001 ; \mathrm{B}$ $=0.01 ; \mathrm{C}=0.3$.

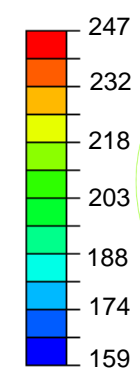

A

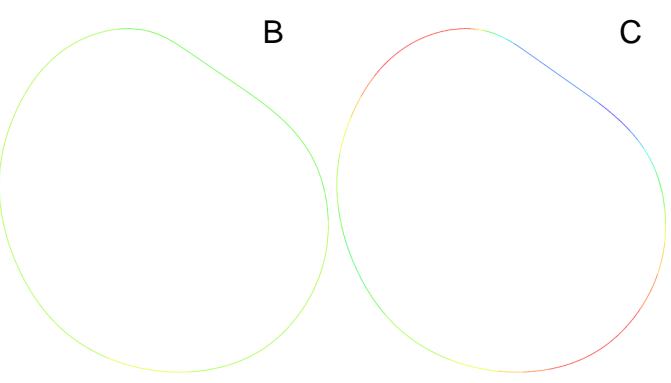

Figure 7 - Tension (in N) of the CS with adjustment of the friction coefficient - Values of the friction coefficients: $\mathrm{A}=0.001 ; \mathrm{B}=0.01 ; \mathrm{C}=$ 0.3 .

MRI scan of the same leg wearing the CS.

Data acquisition One of the subjects (female, 24 years) underwent an MRI scan while wearing a CS in addition to the protocol described previously $[1,2]$. 
Implementation To compare the final geometry predicted by FE to the geometry imaged by MRI, it is first necessary to perform an elastic registration of the MRI scan of the leg without CS as the leg in the MRI scan was deformed due to the contact between the calf and the platform [1]. The different steps of the registration are described below:

1. Rigid registration of both modalities (Figure 8).

2. After segmenting the global contour of the leg US and MRI images, the same anatomic landmarks are selected on each image (Figure 9).

3. The coordinates of selected anatomic landmarks are stored. Then, from each couple of anatomic landmarks, the difference between the coordinates is derived as:

$$
\begin{aligned}
& D x_{i}=x_{i}^{I R M}-x_{i}^{U S} \\
& D y_{i}=y_{i}^{I R M}-y_{i}^{U S}
\end{aligned}
$$

Where $x_{i}^{I R M}$ and $y_{i}^{I R M}$ are the $x$ et $y$ coordinates of selected anatomic landmarks in the MRI scan; $x_{i}^{U S}$ et $y_{i}^{U S}$ are the $x$ et $y$ coordinates of $x$ and $y$ of selected anatomic landmarks in US reconstructions; $D x_{i}$ and $D y_{i}$ are the difference between these coordinates.

4. A linear interpolation is used to derive the difference $D x$ et $D y$ for all the pixels from the difference at the anatomic landmarks (Figure 10 $\mathrm{A}$ and $\mathrm{B})$.

5. The calculated differences in $x$ (Figure $10-\mathrm{A}$ ) and in $y$ (Figure 10 - B) are applied onto the coordinates of each pixel in the US image (Figure 10 - C). Finally, the US image is deformed (Figure 10 - D) and aligned to the MRI image (Figure 10 - E).

Evaluation The geometry of the leg predicted by the patient-specific FE model shows a good agreement with the MRI geometry under elastic compression (Figures 11). In both cases (FE et MRI), the geometry of the leg is rounder when subjected to elastic compression.

\section{Results}

The patient-specific FE model was implemented for the calf of four volunteers. The response of their lower leg to elastic compression was calculated. Here we present the results of these simulations.

\subsection{Distribution hydrostatic pressure}

Without muscle contraction Fig 12 shows hydrostatic pressure maps for the four subjects. The hydrostatic pressure is a meaningful metrics of 


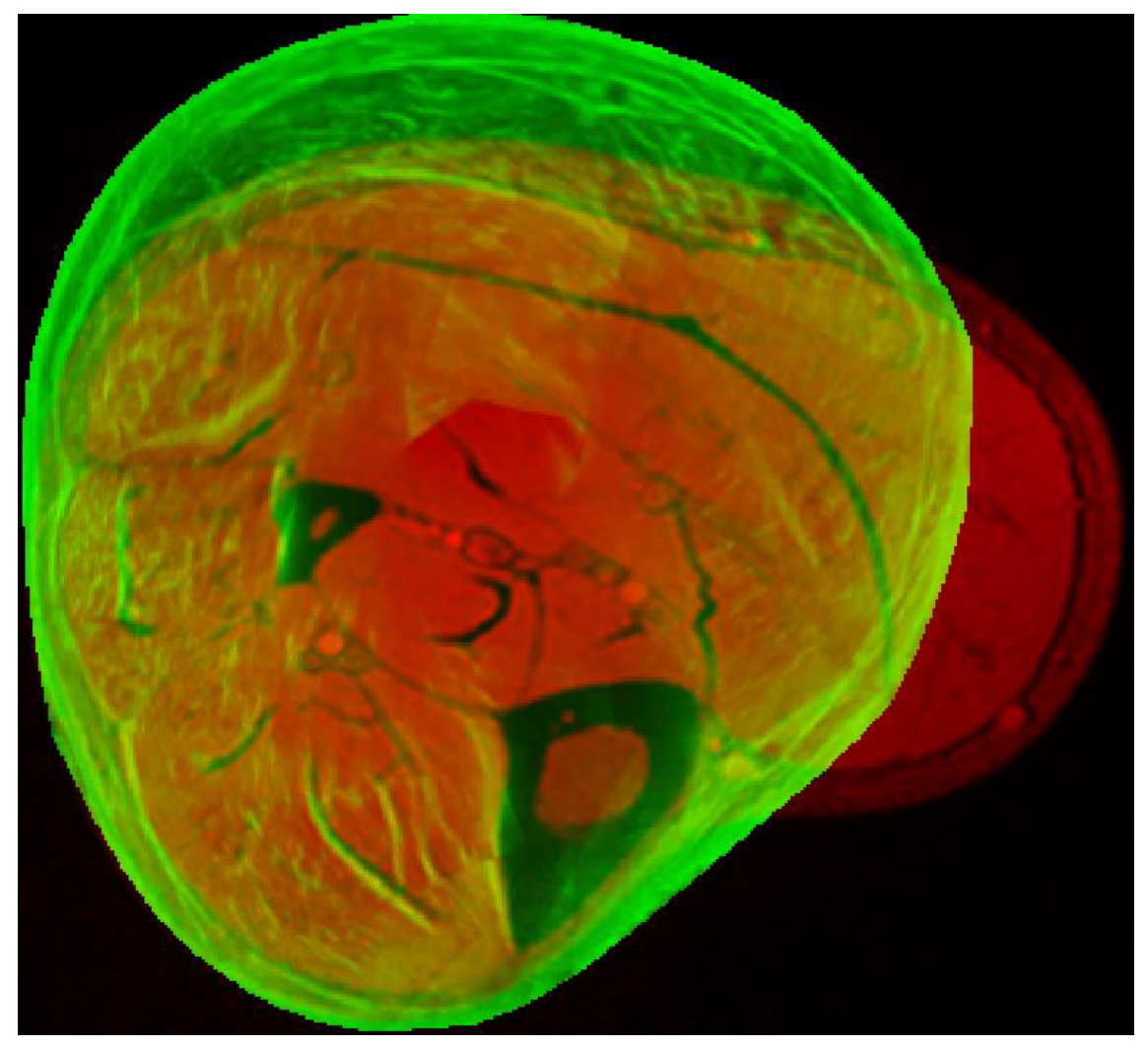

Figure 8 - Rigid-body registration of both modalities (US and MRI).

the effects of elastic compression as it does not depend on the choice of coordinate system and it can be applied to analyze pressure gradients that may indicate possible fluid flows.

With muscle contraction Muscle contraction induces a significant change of the geometry of the calf and also a change of material properties. The geometry of the calf was acquired using US imaging for an active state of muscles for each subject. This active state corresponded to a voluntary contraction equal to about $30 \%$ of the maximum voluntary contraction. The material properties of the fascia cruris and of the skin and fat tissues were also measured using USE in the active state of muscles. Only the material 


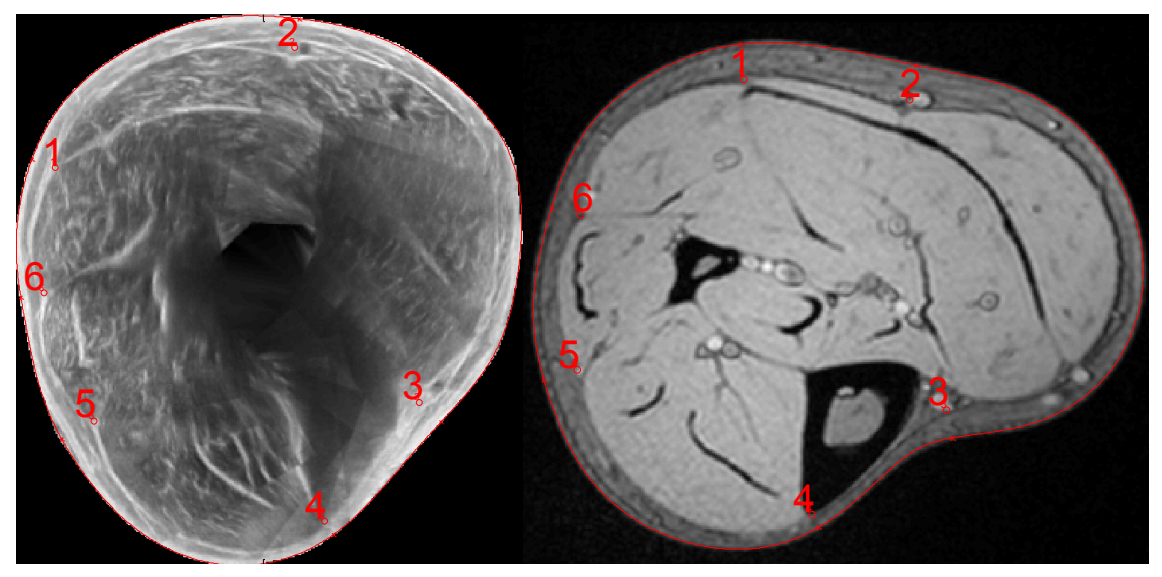

Figure 9 - Selection of anatomic landmarks on the contour of the leg.

properties of deep soft tissues could not be identified for the active state of muscles due to the discomfort induced by the compression test on contracted muscles. Moreover, to our knowledge, the literature does not report material properties of muscles for a contraction equal to $30 \%$ of the maximum voluntary contraction. It was therefore chosen to run a parametric study by varying the elastic parameter $C_{10}$ of deep soft tissues in a range defined as follows, from 18 to $360 \mathrm{kPa}$ ).

Figure 13 shows hydrostatic pressure maps obtained by these simulations for the four subjects. It is observed that the increase in the elastic modulus of the deep soft tissues has an impact on the distribution of the hydrostatic pressure by increasing the tension and compression between the skin and the fascia cruris.

Effects of elastic compression For the four volunteers, the distributions of hydrostatic pressure appear heterogeneous (Figures 12 and 13). The maximum pressure is located where the radius of curvature is lower (near the tibia) and vice versa, the minimum pressures were observed in flat areas.

Substantial variability between subjects is observed in the distribution of these pressures. The maximum pressure range is from $4.1 \mathrm{kPa}$ to $5.3 \mathrm{kPa}$. Local maxima and minima are not located at the same place depending on 

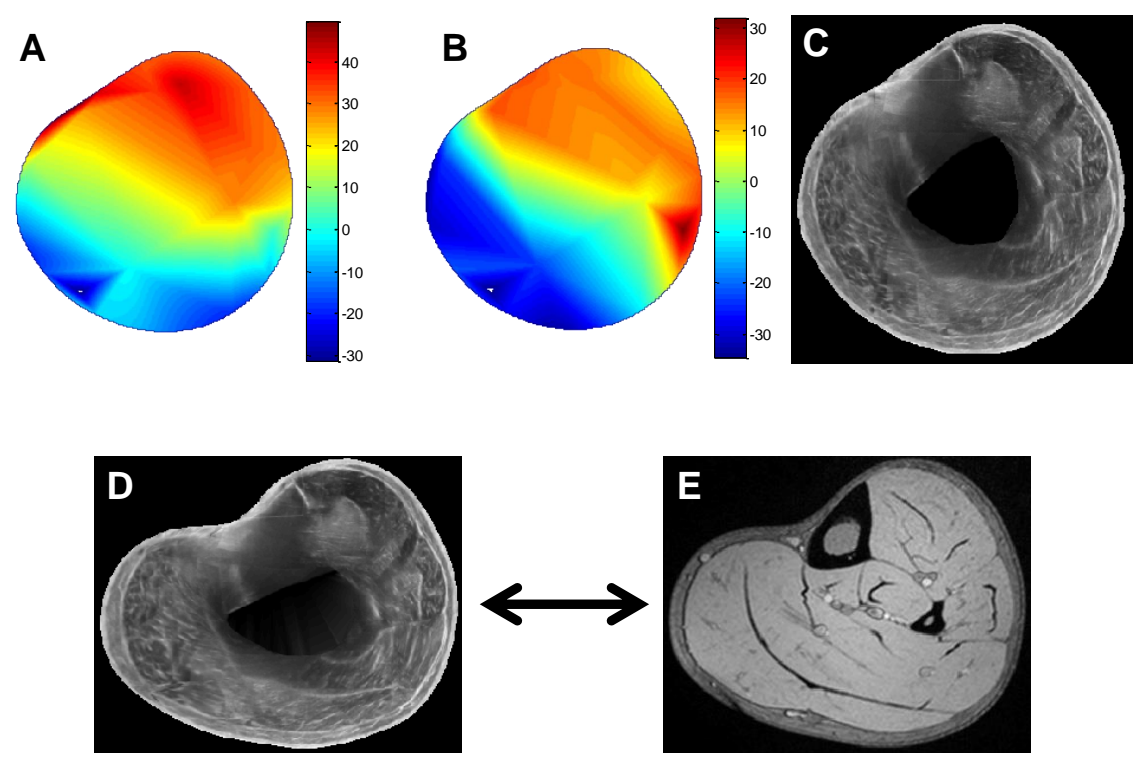

Figure 10 - Linear interpolation of displacements calculated at anatomic landmarks selected by the operator on both modalities. Distributions of displacements in pixels in directions $x(\mathrm{~A})$ and $y(\mathrm{~B})$. These displacements are applied to pixels of image $\mathrm{C}$, transforming it in image $\mathrm{D}$, before aligning it with image $\mathrm{E}$.

the subjects. For example, for subjects 2 and 3 (Figure $13-\mathrm{B}$ and C), a very low pressure is observed at the small saphenous vein located on the posterior side of the leg whereas the pressure on the small saphenous vein for subject 4 appears much larger (Figure $13-\mathrm{D}$ ).

\subsection{Closure of veins}

The percentage of closing for the greater and the small saphenous veins is calculated. The small saphenous vein is located on the posterior side of the leg and the greater saphenous vein on the anteromedial side of the leg (Figure 1). To derive the closure ratio, the coordinates of nodes of the venous lumen are taken at the initial state and at the end of the FE simulation. 

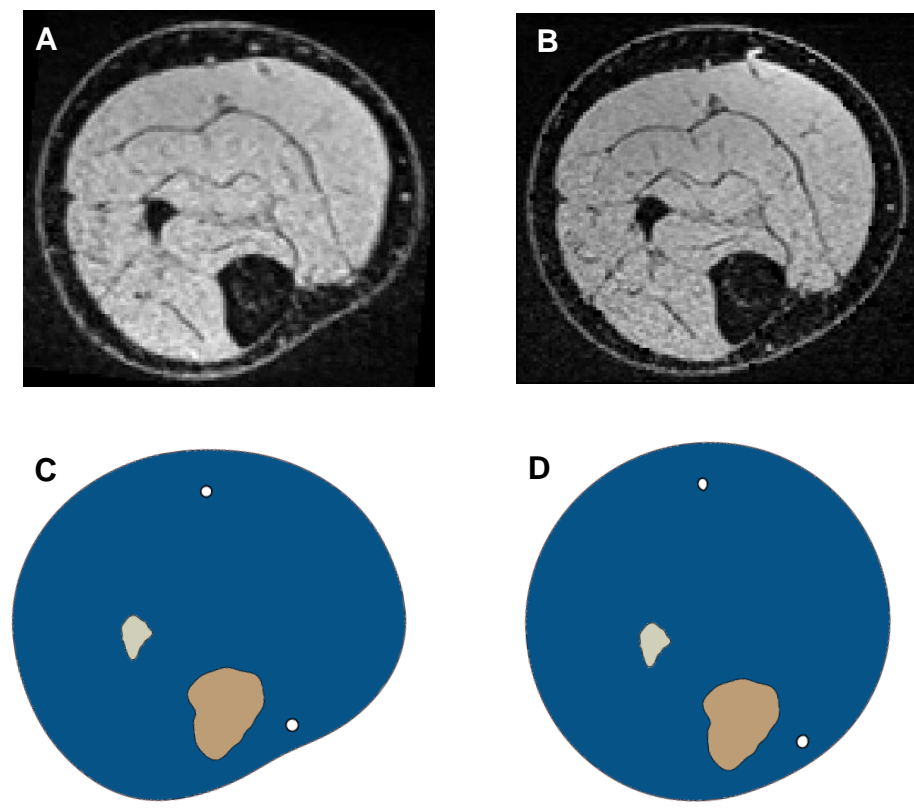

Figure 11 - MRI cross sections of the leg with (B) and without (A) wearing the CS. Initial geometry of the leg in the FE model (C) and FE predictions of the geometry of the leg under elastic compression (D). This comparison was performed for the only subject for whom a MRI scan was available with and without wearing a CS. For the other subjects, alignment of MRI and US modalities was achieved as shown in Figures 8, 9 and 10 but no other MRI validation was possible for numerical predictions of the effects of elastic compression.

Then, the luminal areas of the veins in the initial and final state are calculated to derive the closing percentage.

For each vein of each subject, the closing ratio values are given in Table 2 . In this table, results are given for the inactive muscle state and for the active muscle state. For each vein of each subject in the active muscle state, the closing ratio is also shown in Figure 15. 
A

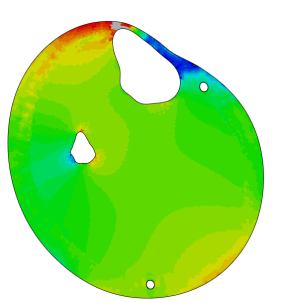

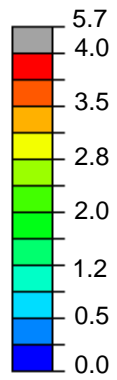
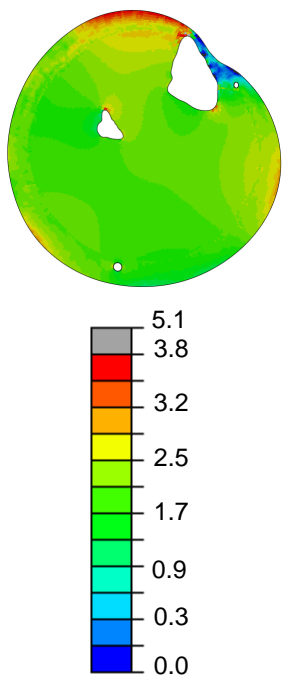

C
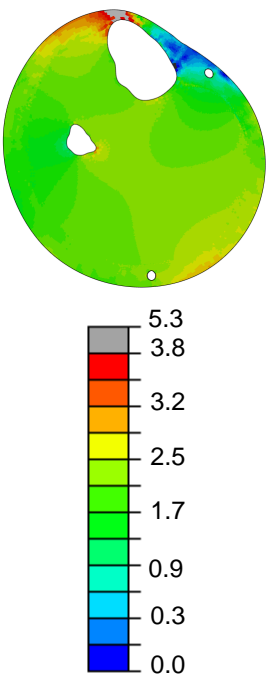

D
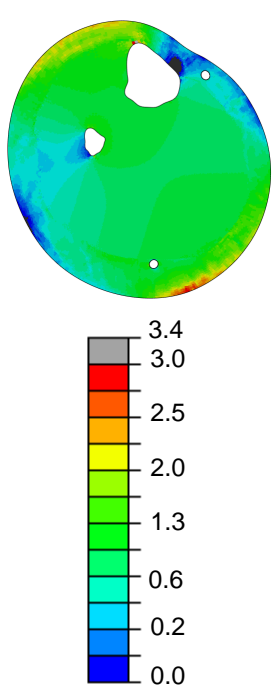

Figure 12 - Distribution of the hydrostatic pressure in $\mathrm{kPa}$ for the right leg of 4 subjects under elastic compression with an applied pressure of about $20 \mathrm{mmHg}$ (A subject 1, B subject 2, C subject 3 et D subject 4).

Influence of muscle contraction In Table 2, we note that the overall vein closure ratios are larger in the inactive state than in the active one. Indeed, in the inactive muscle condition, material properties of superficial soft tissue measured using USE were found smaller, as this is shown in Figure 14) where we report all the results obtained with the USE technique which were previously published [1]. More compliant material properties facilitate the closure of the veins by the elastic compression. For the active muscle condition, we see in Figure 15 for all subjects and for both veins, that the larger the elastic modulus in deep soft tissues, the smaller the closure ratio.

Influence the vein location In Figure 15, the closure ratio of the small saphenous vein is smaller than the one of the greater saphenous vein. This 


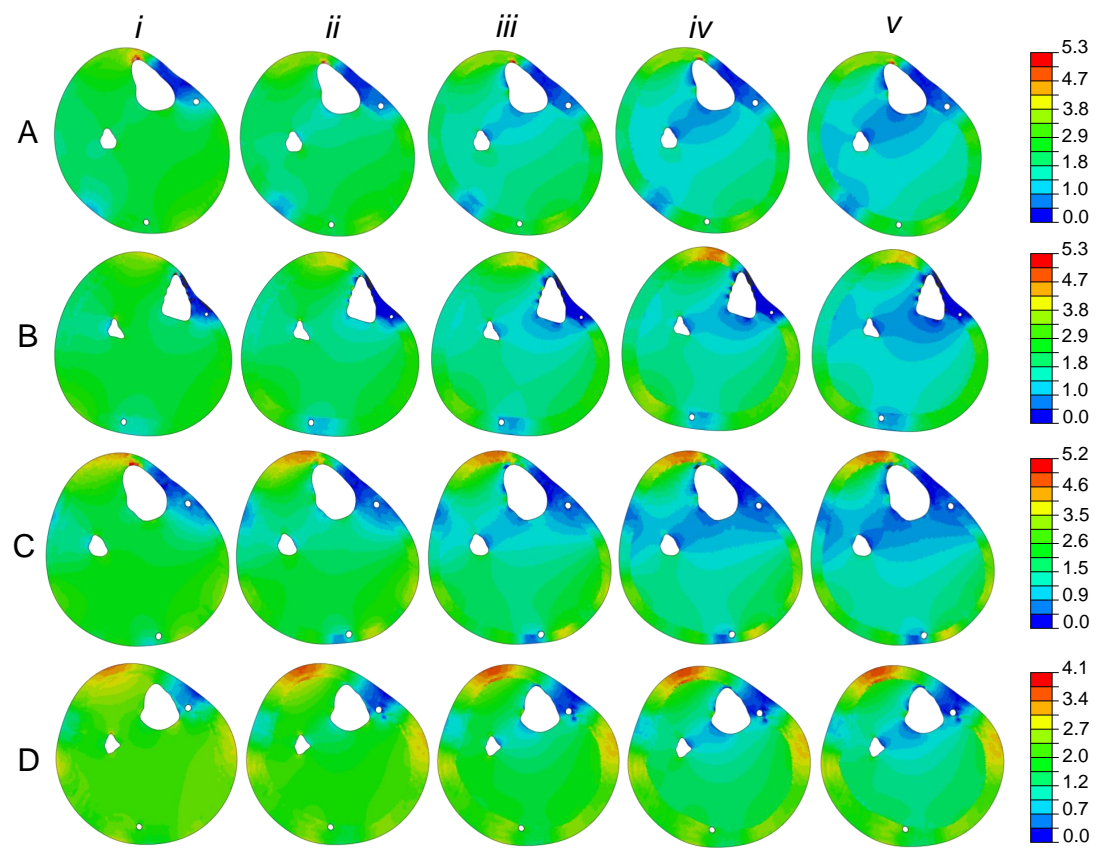

Figure 13 - Distribution of the hydrostatic pressure in $\mathrm{kPa}$ for the right leg of 4 subjects under elastic compression with an applied pressure of about $20 \mathrm{mmHg}$ (A: subject 1, B: subject 2, C: subject 3 and D: subject 4). Below the fascia cruris, $C_{10}$ values are set as follows: $i \rightarrow 18 \mathrm{kPa}$; $i i \rightarrow 72 \mathrm{kPa}$; $i i i \rightarrow 180 \mathrm{kPa} ; i v \rightarrow 288 \mathrm{kPa} ; v \rightarrow 360 \mathrm{kPa}$.

is due to the position of the small saphenous vein which is located on the posterior side of the leg where the curvature is low. In this area mainly composed of soft tissues, the CS applies a smaller pressure. The larger closing ratio of the greater saphenous vein is also due to its position, but more specifically to its proximity with the tibia bone. Indeed, the greater saphenous vein is located on the anteromedial part of the leg along the tibia. Therefore, even though the area is slightly curved, low pressure applied by the CS results in a large closure ratio of the vein. 


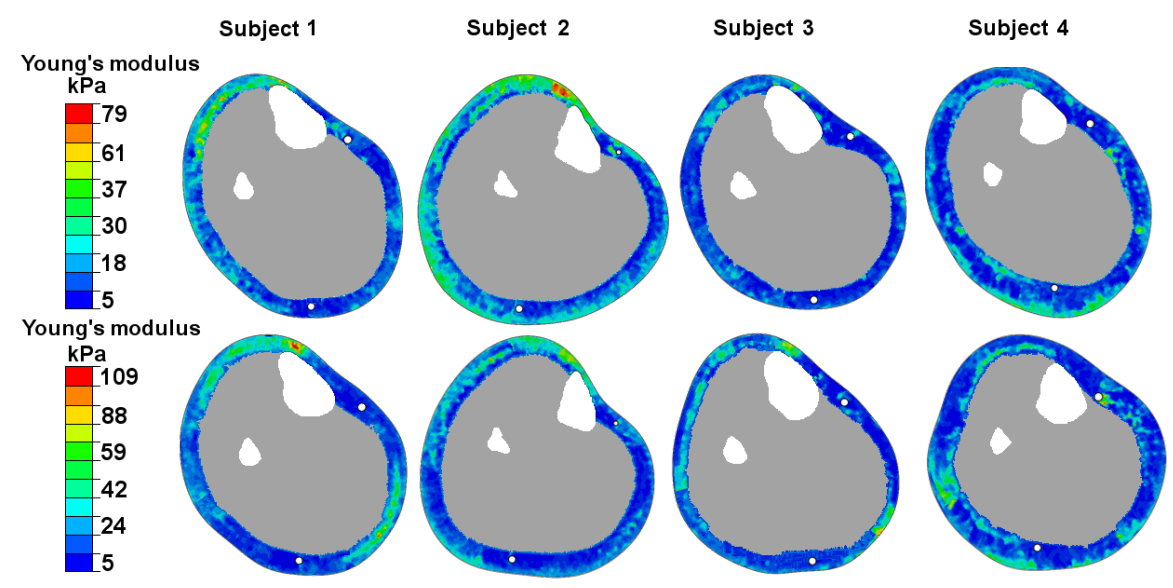

Figure 14 - Distributions of elastic moduli of superficial soft tissues measured using the USE technique - Line 1: inactive muscle condition; Line 2: active muscle condition.

\section{Discussion}

\subsection{Originality of the study}

In a previous study [15], we conducted a parametric numerical study showing that the biomechanical response of the superficial veins to elastic compression depends on three mechanical factors: the height of the cross section leg, the position of the vein on the contour of the leg (radius local curvature) and the mechanical properties of the adipose tissue. This previous study highlighted the specific response of the leg of each subject to elastic compression.

Therefore in the current study, patient-specific FE models, customized in terms of geometry and material properties of the soft tissues, were implemented. These models take into account the heterogeneity of mechanical properties in soft tissues and the effect of muscle contraction.

We compared the hydrostatic pressures obtained with the patient-specific model to hydrostatic pressures obtained with a model having the same geometry but with homogeneous material properties, i.e with no custumization of material properties (Figure 16). This comparison pointed significant local 
Table 2 - Closure ratios of veins under elastic compression for an inactive muscle condition and for an active muscle condition. For the latter, the studied cases correspond to $C_{10}$ values set as follows: $i \rightarrow 18 \mathrm{kPa}$; $i i \rightarrow$ $72 \mathrm{kPa}$; iii $\rightarrow 180 \mathrm{kPa} ; i v \rightarrow 288 \mathrm{kPa} ; v \rightarrow 360 \mathrm{kPa}$.

\begin{tabular}{|c|c|c|c|c|}
\hline \multirow[t]{2}{*}{ Subject } & \multirow[t]{2}{*}{$\begin{array}{l}\text { Muscular } \\
\text { state }\end{array}$} & \multirow[t]{2}{*}{ Case studied } & \multicolumn{2}{|c|}{$\begin{array}{l}\text { Closure of saphenous veins } \\
\text { under elastic compression - \% }\end{array}$} \\
\hline & & & Small & Greater \\
\hline \multirow{6}{*}{1} & inactive & & 7.2 & 17.6 \\
\hline & & $i$ & 4.5 & 14.4 \\
\hline & & $i$ & 3.6 & 11.4 \\
\hline & active & $i i i$ & 3.1 & 10.3 \\
\hline & & $i v$ & 2.9 & 9.9 \\
\hline & & $v$ & 2.8 & 9.8 \\
\hline \multirow{6}{*}{2} & inactive & & 1.9 & 18.8 \\
\hline & & $i$ & 2.8 & 11.5 \\
\hline & & $i i$ & 2.1 & 8.3 \\
\hline & active & $i i i$ & 1.9 & 7.5 \\
\hline & & $i v$ & 1.8 & 7.2 \\
\hline & & $v$ & 1.7 & 6.9 \\
\hline \multirow{6}{*}{3} & inactive & & 7.3 & 17.7 \\
\hline & & $i$ & 3.3 & 11.3 \\
\hline & & $i i$ & 2.1 & 8.8 \\
\hline & active & $i i i$ & 1.7 & 8.0 \\
\hline & & $i v$ & 1.6 & 7.7 \\
\hline & & $v$ & 1.4 & 7.6 \\
\hline \multirow{6}{*}{4} & inactive & & 1.6 & 10.8 \\
\hline & & $i$ & 2.9 & 9.4 \\
\hline & & $i i$ & 1.7 & 5.2 \\
\hline & active & $i i i$ & 1.3 & 3.9 \\
\hline & & $i v$ & 1.1 & 3.5 \\
\hline & & $v$ & 1.1 & 3.3 \\
\hline
\end{tabular}

differences in the response of the tissues to elastic compression. Moreover, the effect of muscle contraction was shown to have a significant impact on the closure of the small and greater saphenous veins. 


\subsection{Prediction of the effects of elastic compression}

We showed that the distribution of superficial pressure, which is felt by peri-nerves, is generally determined by the shape and size of the subject's leg, especially by local radii of curvature. Locally, the consideration of the mechanical properties of soft tissue inhomogeneities in the vicinity of the superficial veins may also explain in part the large inter-individual variability encountered for the efficiency of compression therapy.

The distributions of hydrostatic pressures shown in this chapter confirm the idea that CS are designed to increase the peri-venous pressure, which helps to reduce the transmural pressure of the superficial veins. The transmural pressure is also known to be connected to the tension in the vein wall by following the Laplace law [27]. The reduction of this tension may be an important aspect of the treatment of symptoms associated with venous deficiency.

Several studies $[28,29,30]$ showed that muscle activation is modified by wearing CS. Indeed, skin receptors are involved not only in proprioceptive effects, through Pacini and Rufini mechanoreceptors [31]. These receptors are part of superficial mechanoreceptors in the soft tissues. They may be primarily activated by a static pressure and a stretch [32].

One potential explanation of proprioceptive effects of a compression treatment may be proposed. With hydrostatic pressures approaching the excitability threshold of the mechanoreceptors, these mechanoreceptors could detect smaller tangential movements or lower pressure changes. This could induce slight muscle activation that would participate to a more efficient blood draining in the calf. Future work will focus on a thorough investigation of this assumption.

\subsection{Limitations and future work}

The work presented in this chapter still presents some limitations. Addressing these limitations will require important effort of research on the biomechanics of the calf. We would like to mention the following directions for this effort of research:

1. although the use of $2 \mathrm{D}$ models was shown to be relevant [13], it would be interesting to extend our approach to fully 3D finite-element models of the calf;

2. elastic modulus of the superficial tissues were obtained using ultrasound elastography but the technique could not be used for the deep 
tissues. It would be interesting to implement an elastographic method for the reconstruction of complete fields of material properties both in deep and superficial soft tissues, such as MRE for instance [?];

3. ultrasound elastography is limited to the identification of linear elastic moduli whereas soft tissues have a nonlinear behaviour;

4. compression with about $20 \mathrm{mmHg}$ was analyzed. It would be interesting to investigate the effects of higher pressures which are important for the treatment of specific pathologies of the calf such as lymphodema;

5. the effects of compression should be modelled on a large cohort of subjects in order to establish statistical analyses.

\section{Conclusions}

This chapter presented the combination of two innovative methodologies for the custumization of patient-specific FE model of the calf in terms of material properties. This led to original numerical predictions of the biomechanical effects of elastic compression on the leg which were validated against MRI. Preliminary answers could be found to the inter-subject variability of efficiency encountered during treatment of venous insufficiency by compression therapy. This constitutes an essential step towards the customization of compression therapy in the future.

\section{References}

[1] Fanny Frauziols, Jérôme Molimard, Laurent Navarro, Pierre Badel, Magalie Viallon, Rodolphe Testa, and Stéphane Avril. Prediction of the biomechanical effects of compression therapy by finite element modeling and ultrasound elastography. IEEE Transactions on Biomedical Engineering, 62(4):1011-1019, 2015.

[2] Fanny Frauziols, Fanette Chassagne, Pierre Badel, Laurent Navarro, Jérôme Molimard, Nicolas Curt, and Stéphane Avril. In vivo identification of the passive mechanical properties of deep soft tissues in the human leg. Strain, 2016. submitted.

[3] Pierre Kamina. Anatomie clinique: Tome 1, anatomie généralemembres. Maloine ed, Paris, 1990.

[4] Hermann Braus and Curt Elze. Anatomie des menschen: ein lehrbuch für studierende und ärzte..., volume 1. J. Springer, 1921. 
[5] Jaap van der Wal. The architecture of the connective tissue in the musculoskeletal system-an often overlooked functional parameter as to proprioception in the locomotor apparatus. International journal of therapeutic massage \& bodywork, 2(4):9-23, January 2009.

[6] Helene M Langevin, Kirsten N Storch, Marilyn J Cipolla, Sheryl L White, Thomas R Buttolph, and Douglas J Taatjes. Fibroblast spreading induced by connective tissue stretch involves intracellular redistribution of $\alpha$-and $\beta$-actin. Histochemistry and cell biology, 125(5):487495, 2006.

[7] N J Papadopoulos, M F Sherif, and E N Albert. A fascial canal for the great saphenous vein: gross and microanatomical observations. Journal of anatomy, 132(Pt 3):321-9, May 1981.

[8] David Bergqvist. The Vein Book. 2007.

[9] John J Bergham. The vein book. Elsevier, 2007.

[10] Stéphane Avril, Pierre Badel, Laura Dubuis, Pierre-Yves Rohan, Johan Debayle, Serge Couzan, and Jean-François Pouget. Patient-specific modeling of leg compression in the treatment of venous deficiency. In Amit Gefen, editor, Patient-Specific Modeling in Tomorrow's Medicine, number 09 in Studies in Mechanobiology, Tissue Engineering and Biomaterials, pages 217-238. Springer Berlin Heidelberg, January 2012.

[11] RJ Morris and JP Woodcock. Intermittent pneumatic compression or graduated compression stockings for deep vein thrombosis prophylaxis? a systematic review of direct clinical comparisons. Ann Surg, 2010.

[12] Stéphane Avril, Laura Bouten, Laura Dubuis, Sylvain Drapier, and Jean-François Pouget. Mixed experimental and numerical approach for characterizing the biomechanical response of the human leg under elastic compression. ASME Journal of Biomechanical Engineering, 132(3):031006, March 2010.

[13] Laura Dubuis, Stéphane Avril, Johan Debayle, and Pierre Badel. Identification of the material parameters of soft tissues in the compressed leg. Computer Methods in Biomechanics and Biomedical Engineering, 15(1):3-11, 2012.

[14] Pierre-Yves Rohan, Pierre Badel, Bertrand Lun, Didier Rastel, and Stéphane Avril. Prediction of the biomechanical effects of compression therapy on deep veins using finite element modelling. Annals of Biomedical Engineering, pages 1-11, 2014. 
[15] Pierre-Yves Rohan, Pierre Badel, Bertrand Lun, Didier Rastel, and Stéphane Avril. Biomechanical response of varicose veins to elastic compression: a numerical study. Journal of Biomechanics, 46(3):599603, February 2013.

[16] Hai-Chao Han. A biomechanical model of artery buckling. Journal of biomechanics, 40(16):3672-3678, 2007.

[17] Hai-Chao Han. Blood vessel buckling within soft surrounding tissue generates tortuosity. Journal of biomechanics, 42(16):2797-2801, 2009.

[18] Kas Hibbit. Abaqus theory and user manuals version 6.9. USA: ABAQUS Inc, 2009.

[19] PG Agache, C Monneur, JL Leveque, and J De Rigal. Mechanical properties and young's modulus of human skin in vivo. Archives of dermatological research, 269(3):221-232, 1980.

[20] Gaétan Boyer, Hassan Zahouani, Alain Le Bot, and Leslie Laquieze. In vivo characterization of viscoelastic properties of human skin using dynamic micro-indentation. In Engineering in Medicine and Biology Society, 200\%. EMBS 2007. 29th Annual International Conference of the IEEE, pages 4584-4587. IEEE, 2007.

[21] Alexandre Delalleau, Georges Josse, Jean-Michel Lagarde, Hassan Zahouani, and Jean-Michel Bergheau. A nonlinear elastic behavior to identify the mechanical parameters of human skin in vivo. Skin Research and Technology, 14(2):152-164, 2008.

[22] F.M. Hendriks, D. Brokken, C.W.J. Oomens, D.L. Bader, and F.P.T. Baaijens. The relative contributions of different skin layers to the mechanical behavior of human skin in vivo using suction experiments. Medical Engineering 83 Physics, 28(3):259-266, 2006.

[23] J Jachowicz, R McMullen, and D Prettypaul. Indentometric analysis of in vivo skin and comparison with artificial skin models. Skin Research and Technology, 13(3):299-309, 2007.

[24] Laura Bouten. Identification des propriétés mécaniques des tissus constitutifs du mollet pour l'étude mécanique de la contention. $\mathrm{PhD}$ thesis, Ecole Nationale Supérieure des Mines de Saint-Etienne, March 2009. 
[25] Jarkko T Iivarinen, Rami K Korhonen, Petro Julkunen, and Jukka S Jurvelin. Experimental and computational analysis of soft tissue stiffness in forearm using a manual indentation device. Medical engineering ES physics, 33(10):1245-1253, 2011.

[26] Lutz-Christian Gerhardt, Alexander Lenz, Nicholas D Spencer, Thomas Münzer, and Stephan Derler. Skin-textile friction and skin elasticity in young and aged persons. Skin Research and Technology, 15(3):288-298, August 2009.

[27] Rebecca J Gusic, Matus Petko, Richard Myung, J William Gaynor, and Keith J Gooch. Mechanical properties of native and ex vivo remodeled porcine saphenous veins. Journal of biomechanics, 38(9):1770-1779, 2005.

[28] Dan K Ramsey, Per F Wretenberg, Mario Lamontagne, and Gunnar Németh. Electromyographic and biomechanic analysis of anterior cruciate ligament deficiency and functional knee bracing. Clinical Biomechanics, 18(1):28-34, 2003.

[29] Daniel Théoret and Mario Lamontagne. Study on three-dimensional kinematics and electromyography of acl deficient knee participants wearing a functional knee brace during running. Knee Surgery, Sports Traumatology, Arthroscopy, 14(6):555-563, 2006.

[30] William J Kraemer, Shawn D Flanagan, Brett A Comstock, Maren S Fragala, Jacob E Earp, Courtenay Dunn-Lewis, Jen-Yu Ho, Gwendolyn A Thomas, Glenn Solomon-Hill, Zachary R Penwell, et al. Effects of a whole body compression garment on markers of recovery after a heavy resistance workout in men and women. The Journal of Strength E6 Conditioning Research, 24(3):804-814, 2010.

[31] Roland S Johansson and Åke B Vallbo. Tactile sensory coding in the glabrous skin of the human hand. Trends in Neurosciences, 6:27-32, 1983.

[32] John H Martin and Thomas M Jessell. Modality coding in the somatic sensory system. Principles of neural science, 3:341-352, 1991. 


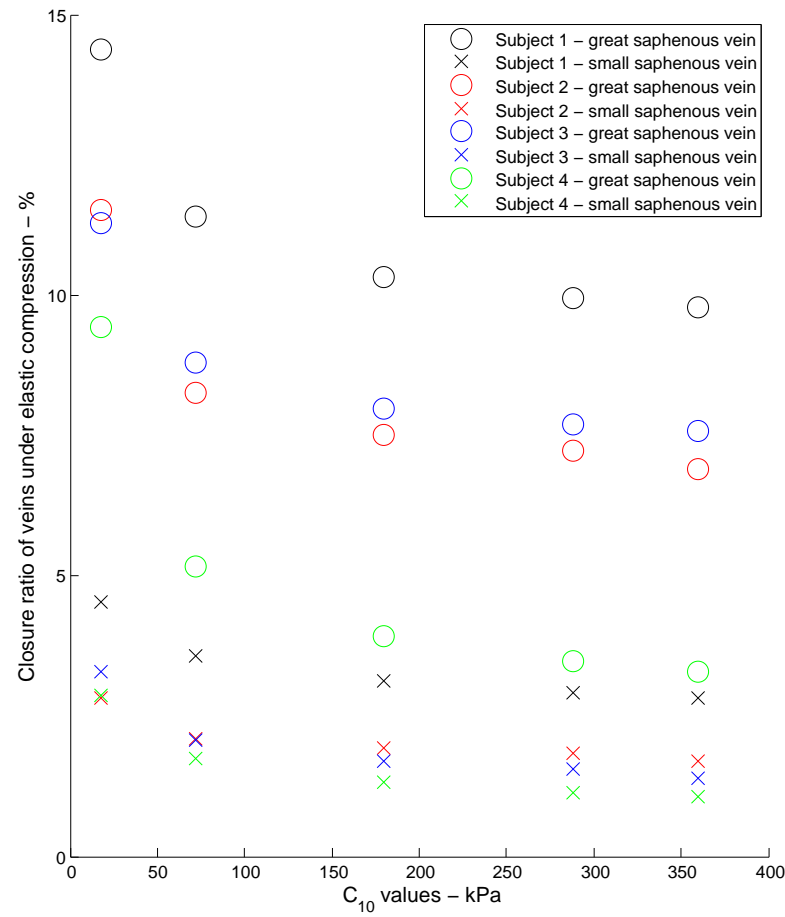

Figure 15 - Closure ratios of veins under elastic compression for an active muscle condition with varying elastic properties of deep soft tissues. 

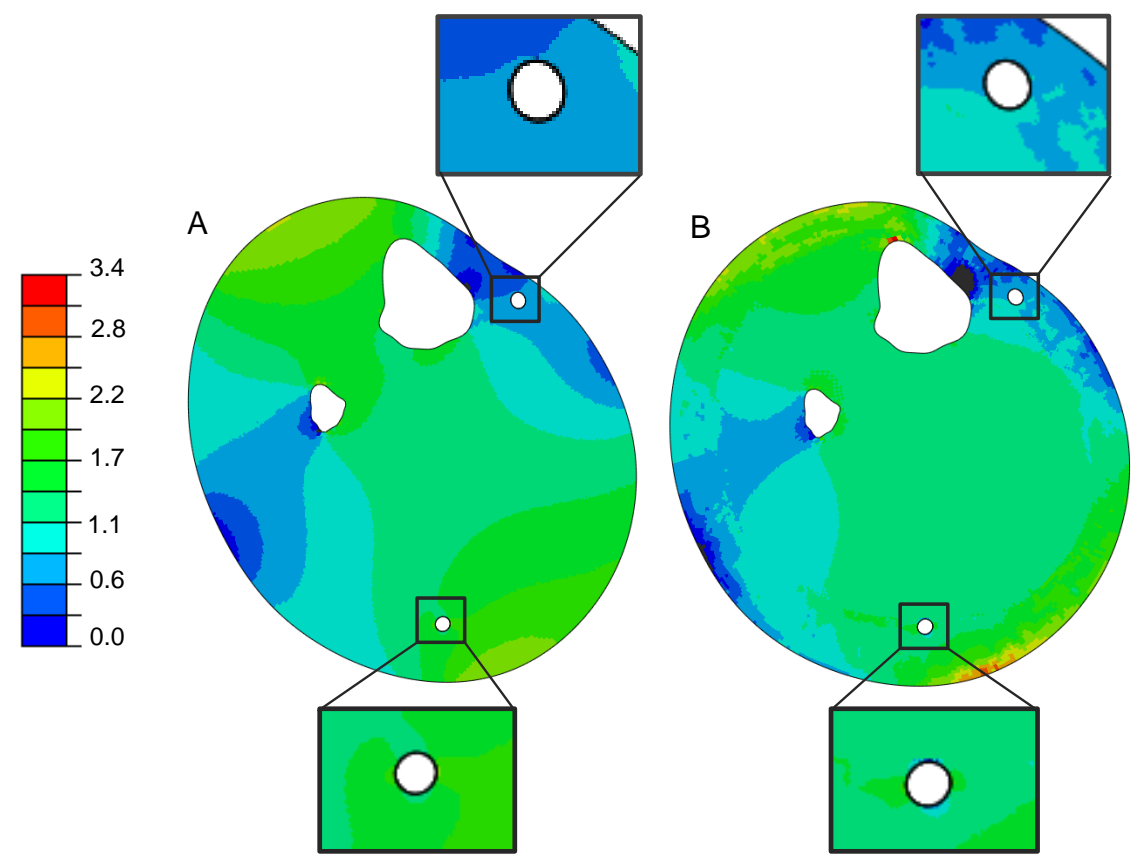

Figure 16 - Distribution of the hydrostatic pressure in $\mathrm{kPa}$ in the calf under elastic compression - A: computational model with no customization of material properties; B: computational model with patient-specific heterogeneous material properties. 